\title{
Foscolo et la tradition italienne dans les écrits de
} Giuseppe Mazzini

Foscolo e la tradizione italiana negli scritti di Giuseppe Mazzini

Foscolo and the Italian Tradition in the Writings of Giuseppe Mazzini

\section{Laura Fournier-Finocchiaro}

\section{OpenEdition}

\section{Journals}

Édition électronique

URL : http://journals.openedition.org/cei/2574

DOI : $10.4000 /$ cei.2574

ISSN : 2260-779X

\section{Éditeur}

UGA Éditions/Université Grenoble Alpes

Édition imprimée

Date de publication : 30 juin 2015

Pagination : 269-283

ISBN : 978-2-84310-289-9

ISSN : 1770-9571

\section{Référence électronique}

Laura Fournier-Finocchiaro, «Foscolo et la tradition italienne dans les écrits de Giuseppe Mazzini », Cahiers d'études italiennes [En ligne], 20 | 2015, mis en ligne le 01 janvier 2017, consulté le 26 mars 2021. URL : http://journals.openedition.org/cei/2574 ; DOI : https://doi.org/10.4000/cei.2574 


\title{
FOSCOLO ET LA TRADITION ITALIENNE DANS LES ÉCRITS DE GIUSEPPE MAZZINI
}

\author{
Laura Fournier-Finocchiaro \\ Université Paris 8 - Vincennes Saint-Denis
}

À Londres, au début du mois de juin I840, Giuseppe Mazzini retrouve chez un libraire londonien les manuscrits d'Ugo Foscolo. Cette date, choisie par Sergio Luzzato pour figurer parmi les entrées du troisième volume de l'Atlante della letteratura italiana', est fondamentale pour l'étude de la fortune de Foscolo auprès des écrivains européens du xix ${ }^{\mathrm{e}}$ siècle, qui seront marqués par les éditions mazziniennes des écrits politiques et critiques de Foscolo. Elle est également capitale pour comprendre d'un côté comment se met en place le panthéon des "gloires littéraires italiennes² ${ }^{2}$ au cours du Risorgimento et de l'autre côté comment se développe en Europe la rhétorique de l'exil, à partir de l'interprétation mazzinienne de l'expérience de Foscolo.

Giuseppe Mazzini a eu un rôle fondamental dans la découverte et dans l'accueil de l'œuvre de Foscolo en Europe à la moitié du xix siècle. Le fondateur de la Giovine Italia a en effet mis au jour des œuvres inédites laissées par Foscolo à Londres lors de son exil : en particulier le commentaire à la Divine Comédie et la Lettre apologétique. Admirateur de Foscolo depuis son adolescence, Mazzini renoue à Londres, vers la fin des années I830, avec son intérêt critique pour les ouvres et les essais de Foscolo et s'engage à rétablir la vérité éthique et politique des déclarations foscoliennes contre l'Italie. La Lettre apologétique servira notamment à Mazzini pour une utilisation idéologique du poète-romancier, dont il pouvait revendiquer l'intégrité et qu'il pouvait présenter à la jeunesse comme un exemple d'harmonie entre pensée et action.

I. S. Luzzatto, "Belli di fama e di sventura», dans S. Luzzatto, G. Pedullà (éd.), Atlante della letteratura italiana, vol. III, D. Scarpa (éd.), Dal Romanticismo a oggi, Turin, Einaudi, 20I2, p. I4O-I48.

2. Cf. E. Irace, Itale glorie, Bologne, il Mulino, 2003. 
Nous étudierons ici comment Mazzini exploite l'œuvre et la pensée de Foscolo pour réaliser son opération de construction du modèle littéraire foscolien comme figure de la "tradition italienne»; nous examinerons les éditions mazziniennes des écrits de Foscolo pour tenter de comprendre leur sens et leur effet sur ses contemporains; et enfin nous ouvrirons des pistes de réflexion sur le mythe du prophète en exil qui se dégage de l'interprétation mazzinienne de Foscolo.

\section{Foscolo père et héros de Mazzini : le modèle littéraire foscolien}

Dès l'adolescence de Mazzini, Foscolo apparaît pour le jeune critique et patriote comme un père et un héros. Dans ses essais critiques de la fin des années 1820 , il théorise le modèle littéraire foscolien pour en faire le chef de file du "courant démocratique» du Risorgimento" .

Dans ses Zibaldoni de jeunesse, le nom de Foscolo revient de façon récurrente, associé à l'idée de l'art comme sacerdoce moral et engagement politique concret ${ }^{4}$. La mise en perspective du rôle de Foscolo dans sa jeunesse est explicitée par Mazzini a posteriori, dans sa biographie romancée publiée sous la forme de Note autobiografiche en I86I. Mazzini y raconte sa découverte du sentiment national lorsqu'il rencontre, dans les rues de Gênes, des insurgés en exil après les moti de I82I. Ce jour-là se forme en lui la pensée qu'il pouvait, voire qu'il devait lutter pour la liberté de sa patrie. Mais il explique que le sens de cet épisode ne lui apparaît clairement que par le biais de la lecture d'un livre, le roman de Foscolo, Ultime lettere di Jacopo Ortis, qui devint à ce moment-là son bréviaire : «L'Ortis che mi capitò allora fra le mani, mi infanatichì : lo imparai a memoria. La cosa andò tanto oltre che la mia povera madre temeva di un suicidios"».

La signification politique de la réalité perçue par le jeune Mazzini, qui frappa profondément son imagination, n'est comprise que par l'intermédiaire d'un roman qui donne à l'expérience de l'exil toute son épaisseur de drame humain et politique. Mazzini veut donc nous faire penser et croire

3. Mazzini renvoie fréquemment à l'exemple de Foscolo : "gli scritti d'Ugo Foscolo, anima deliberata, e possente» (G. Mazzini, Prose di Salvatore Betti [1828], dans Scritti editi ed inediti, Imola, Galeati, I906-1943, vol. I, p. 90); «Ugo Foscolo, per acume d'ingegno, filosofia di pensiero, e potenza d'espressione a null'altro secondo; per nobiltà di cuore, e indipendenza di vita, primo" (G. Mazzini, Necrologia — Vincenzo Monti [I828], dans Id., Scritti, ouvr. cité, vol. I, p. Io8).

4. Les maîtres des jeunes Génois sont plus généralement les "préromantiques». Cf. E. Passerin d’Entrèves, "Il romanticismo "progressivo" di Giuseppe Mazzini negli scritti giovanili (I829-1843)", Bollettino storico pisano, I964-I966, nº 33-35, p. 539-549.

5. G. Mazzini, Note autobiografiche (I86I), dans Id., Scritti, ouvr. cité, vol. LXXVII, p. 9. 
que ce furent ses lectures à caractère littéraire qui permirent d'allumer sa passion politique : les proses et les vers qui parlaient d'Italie et de liberté. Dans ses notes, Mazzini dressait constamment des listes des protagonistes des lettres italiennes, auteurs de "compositions anciennes et modernes de goût romantique», dans lesquelles il plaçait Dante, Giacomo da Lentini, Guittone, puis Foscolo, Berchet, Brofferio, Pindemonte, Manzoni.

Dans ses articles pour le journal l'Indicatore genovese, Mazzini offre une image claire du sens qu'il attribuait à la littérature, comme engagement éthique et civil et comme moyen d'éducation pour forger la conscience nationale. Il célèbre en particulier les grands poètes : Alfieri, Foscolo, mais aussi le "poète classique» Monti, car d'après lui les grands hommes, doués de "virtù » et d'intelligence, constituaient la force de la nation. L'Italie devait garder en mémoire et célébrer ses morts illustres, et en cela il s'opposait à la mise en cause par Foscolo du rôle des critiques littéraires : au contraire, Mazzini défendait et prônait une critique constructive, dont le rôle était de préparer le chemin au génie, en recréant des conditions d'harmonie entre l'écrivain et son public, entre le poète et son époque ${ }^{6}$. Mazzini ne cachait pas son admiration pour l'expérience du Conciliatore, qu'il aurait souhaité poursuivre.

Après la suppression de l'Indicatore genovese, Mazzini poursuit temporairement ses publications littéraires dans l'Indicatore livornese, où il peut plus librement donner cours aux déclarations nationales et patriotiques. Il peut alors parler «explicitement et sans voile» de ses tendances politiques et de Foscolo : «Parlammo del Foscolo, al quale, tacendo degli altri meriti, gli italiani devono riverenza eterna per avere egli primo cogli atti e gli scritti rinvigorito a fini di patria il ministero del letterato ${ }^{7}$ ".

Mazzini explique par exemple que c'est grâce au poète que «la bigoncia nazionale suonava una volta ancora di accenti patrii e vigorosi ${ }^{8} »$. Mazzini célèbre le rôle de Foscolo, mais aussi celui de Pietro Giannone et Giovanni

6. Mazzini attribue à l'activité critique une fonction très ample et active, d'aiguillon du progrès historique de la civilisation : "la Critica sola può imprendere l'opera di rinnovamento; e alla Critica ben intesa e trattata come conviensi spetta il duplice ufficio di rieducare un popolo al Genio e il Genio a una fede: due condizioni senza le quali non è possibile Letteratura" (G. Mazzini, Del dramma storico [1830], dans Id., Scritti, ouvr. cité, vol. I, p. 325-326). Cette conception aura d'importantes répercussions sur la littérature des décennies suivantes. Le critique est selon Mazzini l'«éducateur littéraire»; il peut être même le précurseur du poète de l'avenir, traçant le chemin sur lequel procèdera la poésie démocratique moderne. Plus tard, dans son avis aux lecteurs de la revue L'Italiano (I836), Mazzini va jusqu’à affirmer : «il ministero della critica assume aspetto e importanza di sacerdozio" (G. Mazzini, Prefazione d'un periodico letterario [L'Italiano], dans Id., Scritti, ouvr. cité, vol. VIII, p. 82).

7. G. Mazzini, Note autobiografiche, ouvr. cité, p. I4.

8. G. Mazzini, Orazione di Ugo Foscolo a Bonaparte (I829), dans Id., Scritti, ouvr. cité, vol. I, p. I68. 
Berchet : l'attraction de Mazzini pour les écrivains de «l'école démocratique» apparaît déjà clairement ${ }^{9}$.

Après son départ en exil, commence une nouvelle phase critique plus philosophique de Mazzini, qui a été qualifiée par Anna Teresa Ossani de "socialisme humanitaire ${ }^{\mathrm{IO}}$ » : Mazzini élargit ses intérêts sociaux, sous l'influence de Lamennais et Sand, tandis qu'il attaque les partis politiques, les institutions, les modes, et en particulier la nouvelle école littéraire du réalisme.

Au cours des années i830, par ses déplacements et séjours en France, en Suisse et en Angleterre, Mazzini devient pleinement européen et peut élaborer sa pensée politique originale en prenant appui sur différentes réalités et en se confrontant aux intellects de toutes les nations ${ }^{\text {II }}$. Il continue, parallèlement à ses activités politiques (fondation de la Giovine Italia en I832 à Marseille, puis de la Giovine Europa en I834 à Berne), de consacrer une bonne partie de son temps au journalisme et aux études littéraires. Il développe sa doctrine politique et philosophique, mais aussi ses réflexions sur la littérature et l'art, qu'il souhaite animés d'une intention et d'une extension cosmopolites, afin de réaliser son projet de littérature européenne, prélude d'une plus grande association des intellects européens $^{12}$. Il publie d'ailleurs ses réflexions critiques les plus originales au moment même où il formule ses programmes de la Giovine Italia et de la Giovine Europa. Dans le champ littéraire, il précise dans ses essais l'opposition entre poésie individualiste et nécessité d'un art social ${ }^{13}$. Les critiques ont souvent reproché à Mazzini de subordonner ses opinions de critique littéraire à sa propagande politique ${ }^{14}$; mais au contraire, sa définition des aspects politiques de la communauté nationale se prolonge nécessairement chez lui dans des réflexions sur les nations d'un point de vue culturel.

9. F. L. Mannucci, Giuseppe Mazzini e la prima fase del suo pensiero letterario: l'aurora d'un genio, Milan, Casa editrice Risorgimento, I919.

Io. A. T. Ossani, Letteratura e politica in Giuseppe Mazzini, Urbino, Argalia, I973, p. I46.

II. Sur la dimension européenne de la pensée mazzinienne, voir S. Mastellone, Il progetto politico di Mazzini (Italia-Europa), Florence, Olschki, I994 et J. Y. Frétigné, Giuseppe Mazzini: il pensiero politico, Florence, Centro editoriale toscano, 2009.

I2. Cf. les textes recueillis dans l'anthologie de P. M. Sipala, D'una letteratura europea e altri saggi, Fasano, Schena, I99I et L. Fournier-Finocchiaro, "Il pensiero letterario di Giuseppe Mazzini tra "letteratura nazionale" e identità europea", Bollettino della Domus mazziniana di Pisa, a. LIII, 2008, no I-2, p. 7-20.

I3. Que Mazzini nomme «l'arte popolo; l'arte prete; l'arte religione», dans G. Mazzini, Dell'arte in Italia (I835), dans Id., Scritti, ouvr. cité, vol. VIII, p. 54. Tous les arts doivent contribuer au progrès providentiel de l'humanité; mais ils ne pourront atteindre leur réalisation que lorsque la fondation de la patrie créera pour l'artiste les conditions de liberté indispensables; avant ce moment l'artiste est un esclave qui cède à la fatalité et se fait matérialiste.

I4. Depuis les jugements de F. De Sanctis, Mazzini e la scuola democratica (1873-1874), C. Muscetta, G. Candeloro (éd.), Turin, Einaudi, I95I. 
En Angleterre notamment, Mazzini souhaite faire comprendre aux Anglais (et au reste du public européen) la situation réelle de l'Italie, ce qu'il fait en partant de la culture, avec ses portraits de la littérature et de l'histoire contemporaine. Dans son essai Italian Literature since I830 (traduit par Moto letterario in Italia), publié en I837 sur la Westminster Review de John Stuart Mill, Mazzini dépeint la scène littéraire italienne en mettant en évidence sa grande vitalité et son effervescence intellectuelle. Le patriote italien souhaite élargir l'attention du public, jusque-là limitée aux œuvres de Manzoni et Pellico, aux autres grands auteurs contemporains : en particulier Berchet, Guerrazzi et Mameli, qui devaient être reconnus pour la passion qui animait leurs œuvres et pour leur patriotisme «viril», face à la résignation et à la passivité catholique de Manzoni et Pellico ${ }^{\text {I5 }}$. Par la description de la vie littéraire et de ses protagonistes, Mazzini exprimait le désir que l'Italie fasse entendre sa voix et sa volonté patriotique - et qu'elle ait pleinement le droit de la faire entendre.

L'essai Italian Literature since I830 trace un portrait clair et convaincant de la vie littéraire en Italie dans la première phase du Risorgimento. Mazzini distingue deux écoles littéraires principales, qui défendent deux tendances opposées : d'un côté l'école manzonienne, de l'autre l'école foscolienne-byronienne. La préférence de Mazzini se porte nettement sur cette dernière, qu'il qualifie positivement de "nationaliste», audacieuse et combative ${ }^{16}$, et dont le meilleur représentant à l'époque est selon lui le romancier Francesco Domenico Guerrazzi. Mazzini insiste particulièrement sur la fonction sociale de la littérature et sur les premières décennies du $\mathrm{xIx}^{\mathrm{e}}$ siècle comme époque de transition, destinée à être dépassée. Le modèle littéraire de Foscolo lui permet de célébrer la splendeur révolutionnaire et romantique initiale, de par son idée nationale et son amour pour l'histoire et pour Dante, si bien qu'au final : "Con tutta la vita, col concetto ch'ei [Foscolo] si formò della missione poetica, colla guerra inesorabile sostenuta contro quanti la contaminavano di venalità o d'impostura - ei rialzò la Letteratura scaduta e rifece morale l'Arte e l'artefice ${ }^{17}$ ».

Mais ensuite Mazzini souligne l'attrait de l'art romantique pour l'individualité contre la collectivité et montre son insuffisance face aux attentes d'une nouvelle littérature «sociale».

I5. G. Mazzini, Moto letterario in Italia (I837), dans Id., Scritti, ouvr. cité, vol. VIII, p. 358-363.

16. Ibid., p. 363-364 : «La parola Nazione è scritta sulla loro bandiera, e parola d'ordine è ad essi lotta perenne $[. .$.$] contro l'oppressione domestica e l'influenza straniera, contro il mondo intero, contro Dio stesso,$ qualunque volta Dio sembra proteggere, tollerandolo, il male che intorno ad essi trionfa».

17. Ibid., p. 357-358. 
Mazzini cueille ici les lignes directrices du débat littéraire risorgimental et fournit les schémas interprétatifs de la littérature du XIX ${ }^{\mathrm{e}}$ siècle aux historiographes de la littérature. Sa partition est notamment reprise par la suite par Paolo Emiliani Giudici, Luigi Settembrini et Francesco De Sanctis, jusqu'à ce que ce dernier, en distinguant à son tour l'école libérale de l'école démocratique, mette Mazzini lui-même et non plus Foscolo à la tête de la deuxième école. Or, dans la pensée de Mazzini, aucune de ces deux écoles ne correspond pleinement aux attentes de son époque : le patriote italien a pris ses distances de l'école démocratique, dont il situe les initiateurs (Byron et Foscolo) avant sa propre génération : il la juge non seulement trop attirée par le scepticisme et le désespoir, mais surtout déjà dépassée. Ses personnages sont selon lui trop exceptionnels, trop gigantesques, et ses auteurs sont "de puissantes individualités qui font de grands efforts pour maintenir la tradition littéraire, qui font briller de temps en temps tout l'éclat de la pensée poétique» mais, selon Mazzini, «nous n’y voyons que des efforts isolés, solitaires, s'adressant à un petit nombre d'élus, ne tirant rien du peuple et ne lui donnant rien ${ }^{18}$ ».

Bien que critiqué pour ses limites devant être dépassées, le modèle littéraire foscolien a néanmoins fourni à Mazzini l'idée fondamentale de littérature-action ainsi que les éléments de valorisation d'une «littérature de proscrits». Mazzini a en particulier contribué à valoriser le rôle du poeta-vate défini par Alfieri et incarné par Foscolo. Il a surtout donné un programme et une mission, ainsi qu'un certain style, aux écrivains démocrates, notamment aux jeunes volontaires qui participeront aux batailles républicaines et mazziniennes de I848-I849 ${ }^{19}$.

\section{«Enseigner à la jeunesse italienne le culte des morts » : les éditions mazziniennes des écrits de Foscolo}

Le désœuvrement et l'inaction forcée des premiers temps de Mazzini à Londres lui permettent de trouver le temps de travailler sur le passé littéraire italien et surtout de s'intéresser à deux figures qui ont toujours

I8. G. Mazzini, Dello stato attuale della letteratura (I837), dans Id., Scritti, ouvr. cité, vol. XXI, p. 5 (en français dans l'original).

19. Cf. M. Biondi, "L'incorrotto ideale. Mazzini nella tradizione letteraria», dans Id., La tradizione della patria, vol. I : Letteratura e Risorgimento da Vittorio Alfieri a Ferdinando Martini, Rome, Edizioni di Storia e Letteratura, 2009, p. 35-99; L. Fournier-Finocchiaro, "Mazzini inspirateur des lettres italiennes», PRISMI, $\mathrm{n}^{\mathrm{o}}$ II : Ippolito Nievo et le Risorgimento émancipateur, E. Chaarani-Lesourd (éd.), Éditions Chemins de tr@verse, 20I3, p. 333-348. 
été fondamentales pour lui : les poètes Dante Alighieri et Ugo Foscolo. Mazzini construit le mythe des pères de la patrie dans ses études de ces deux figures de "génie», auxquelles il prête des sentiments nationaux.

L'intérêt de Mazzini pour l'œuvre poétique, romanesque et politique de Foscolo reste très vif et ne s'assombrit pas, malgré leurs différences idéologiques et la diversité de leurs matrices culturelles. Mazzini admire avant tout «l'âme italienne" de Foscolo et la façon dont il a contribué à diffuser l'idée nationale dans la péninsule. On sait par exemple que Mazzini possédait dans sa jeunesse une édition originale imprimée du Discorso su la Italia, rédigé par Foscolo à Gênes à la date du «I 8 vendémiaire an VIII», adressé au général français Championnet pour qu'il libère l'ensemble de la péninsule et qu'il proclame la République italienne. Mazzini, partira à la chasse de cet imprimé pendant son exil à Londres, car il le considérait comme un texte fondateur du républicanisme risorgimental qu'il fallait faire mieux connaître ${ }^{20}$.

Après son arrivée à Londres, Mazzini déclare souhaiter reprendre sa rédaction d'une biographie de Foscolo ${ }^{2 \mathrm{I}}$, car c'est d'après lui le meilleur écrivain moderne qui ait su combiner le talent littéraire et l'engagement politique. Mazzini se rendait tous les jours sur sa tombe, il fréquentait les rédactions de journaux à la recherche d'articles, de travaux inédits et de lettres ${ }^{22}$, et recueillait des informations et des nouvelles de ceux qui avaient connu et fréquenté l'exilé mort dix ans auparavant (Giovita Scalvini, Federico Borgno, Giovanni Gaspare Orelli...), dont le souvenir était encore très présent ${ }^{23}$. Déjà avant son arrivée à Londres, Mazzini, impliqué dans le projet d'une édition parisienne des écrits de Foscolo qui ne sera pas réalisée ${ }^{24}$, avait demandé des informations au libraire londonien William Pickering sur le matériel laissé par le poète mourant. Dans son tableau des lettres italiennes de I837, il se plaint encore de l'absence d'une édition complète des œuvres foscoliennes : "Molti suoi lavori rimangono inediti. Due terzi delle sue fatiche su Dante giacciono nella polvere degli scaffali

20. Cf. sa lettre à Lisette Mandrot du 23 septembre I837 dans G. Mazzini, Epistolario, vol. VI (Id., Scritti, ouvr. cité, vol. XIV), p. 99.

2I. Mazzini avait rencontré le frère de Foscolo, Giulio, en septembre I836 en Suisse, et il lui avait promis d'écrire la Vita del Foscolo peu avant son suicide; mais son départ de la Suisse détourna ses pensées.

22. Déjà lors de son séjour parisien, on lit dans sa correspondance les demandes répétées de Mazzini à sa mère pour qu'elle presse Filippo Ugoni de lui retrouver et lui envoyer tous les textes publiés et inédits de Foscolo. Cf. G. Mazzini, Epistolario, vol. V (août I836-1837), p. 4-5, 31-32, I46-I47. Ces demandes redoublent après son arrivée à Londres.

23. Pour une reconstruction des dernières années de la vie de Foscolo en exil et une réflexion sur les motivations qui poussent Mazzini à renoncer à l'écriture d'une biographie de Foscolo, voir G. Gazzola, "A False Edition of the "Comedy", and its Truth", Forum italicum, 2013/2, p. 299-323.

24. Cf. A Linaker, La vita e i tempi di Enrico Mayer, vol. II, Florence, G. Barbèra, I898, p. 2 I. 
del libraio Pickering. Le vite che s'hanno di lui somigliano più ch'altro [a] libelli ${ }^{25}$ ».

Finalement, dans les archives acquises par Pickering que Mazzini retrouve à Londres, il tombe en particulier sur une petite malle remplie de textes non publiés : des brouillons, des articles et surtout la Lettera apologetica, dans laquelle Foscolo affrontait des questions politiques ignorées par les éditeurs italiens. Mazzini annonce à ses correspondants cette trouvaille comme «lettera importantissima per aneddoti della propria vita politica e discolpe da certe accuse ch'essa contiene, e una delle migliori cose, quanto allo stile, che Foscolo abbia scritto in prosa: energica, pura, solenne, sentita $^{26} »$. Dans le texte, Foscolo donnait en effet une légitimation morale à l'exil, se définissant comme "il primogenito profugo», esquissait la perspective d'une rénovation spirituelle de l'Italie, guidée par l'élite des proscrits fuoriusciti, et il construisait un lien de nature héroïque entre l'ambition littéraire et l'élaboration de projets politiques ${ }^{27}$. Mazzini transforme néanmoins cet autoportrait de Foscolo en une ouvre militante, lui permettant de bénéficier du sceau de l'autorité de Foscolo pour justifier sa propre perspective politique et culturelle, qu'en I84O il souhaitait proposer au parti des exilés, vu l'insuccès de l'option révolutionnaire à court terme.

La Lettera était de loin ce qui intéressait le plus Mazzini, qui explique clairement qu'il voulut dans un premier temps n'acquérir que celle-ci. Face au refus de Pickering, c'est pour elle qu'il dut peiner pour acheter et compléter le commentaire dantesque. Le libraire, en effet, "fatto ingordo dalla mia premura, ricusava cederle s'io non comprava il lavoro sul testo dantes $\mathrm{CO}^{28}$ ». Mazzini décida ensuite d'attendre la meilleure opportunité pour lui donner de la visibilité et du poids, en la séparant du travail dantesque. Il n'eut jamais le temps en revanche de compléter sa biographie de Foscolo.

Le volume des Scritti politici inediti di Ugo Foscolo est publié en I844 à Lugano, chez l'éditeur Ruggia. La préface s'ouvre avec le souvenir de la découverte de Foscolo pendant les années inquiètes de sa jeunesse : Mazzini affirme qu'il appréciait plus l'homme que l'écrivain, et en particulier la loyauté de Foscolo, sa franchise, l'audace indomptée de celui qui osait parler haut et fort en face de Napoléon. Il le définissait «più

25. G. Mazzini, Moto letterario, art. cité, p. 358.

26. G. Mazzini, A Q. Mocenni Magiotti (I8 juillet I840), dans Id., Epistolario, vol. IX (Scritti, ouvr. cité, vol. XIX), p. 197.

27. Cfr. G. Nicoletti, Introduzione, dans U. Foscolo, Lettera apologetica, Turin, Einaudi, I999.

28. G. Mazzini, Note autobiografiche, ouvr. cité, p. 264. 
ch'emancipato emancipatore ${ }^{29}$ ", et ce comportement disposait Mazzini à l'indulgence vis-à-vis du sensualisme de Foscolo et de ses opinions généralement contraires aux siennes, et même vis-à-vis de ses exclamations de désespoir pour la patrie. Il préférait rappeler que Foscolo «serbò incorrotta, immutata davanti al potere, davanti alla prospera e all'avversa fortuna, e all'esilio e alla fame, l'indipendenza dell'animo e del pensiero, e riconsacrò a sacerdozio in Italia l'Arte, scaduta pur troppo, salve poche eccezioni, a mestiere ${ }^{30}$ ".

Foscolo lui avait appris l'idée fondamentale qui l'avait guidé pendant sa vie : «la connessione delle lettere col vivere civile e l'indipendenza da tutte autorità fuorché dall'eterna natura e dal Genio ${ }^{3 \mathrm{I}} »$. Mazzini contribuait ainsi à fournir la plus forte idéalisation romantique du personnage de Foscolo. Le patriote indiquait les germes de renouveau présents dans les œuvres de Foscolo, et s'insurgeait contre son biographe Giuseppe Pecchio, trop cancanier, ainsi que contre les diffamations de Tommaseo. Il résumait les principales accusations adressées au poète et refusait de discuter les insinuations calomnieuses. Il désirait au contraire fournir une analyse approfondie de la Lettera apologetica, qui permettait de comprendre, selon Mazzini, "l'âme foscolienne», c'est-à-dire sa pratique politique et sa morale exemplaire. Mazzini avait l'ambition d'harmoniser le culte pour Foscolo avec une évaluation exacte de ses idées inspirées par le sensualisme, mais qui, selon Mazzini, avait été acceptées par le poète plus par un mouvement de caractère que par réflexion posée : «Le opinioni scettiche o disperate che s'incontrano nelle sue pagine prorompono subitanee, come getti di passione impaziente e senza conforto, non come frutto di sistema filosofico meditato lungamente e logicamente ${ }^{32}$ ».

Par la publication des écrits politiques et son projet de biographie, Mazzini souhaitait indiquer aux jeunes patriotes la façon d'intégrer la culture démocratique de la première moitié du siècle, différente par bien des aspects, mais néanmoins encore présente dans le projet politique et culturel des patrioti. On remarque que sa volonté de faire du poète un modèle de comportement entraîne inévitablement une diminution des composantes révolutionnaires de la pensée de Foscolo à des traces résiduelles et non essentielles.

29. G. Mazzini, Articolo premesso agli scritti politici di Ugo Foscolo (I844), dans Scritti, ouvr. cité, vol. XXIX, p. 160.

30. G. Mazzini, Commento foscoliano alla "Divina Commedia» (I842), dans Id., Scritti, ouvr. cité, vol. XXIX, p. 46.

3I. G. Mazzini, Articolo, art. cité, p. I6I.

32. Ibid., p. I77. 
L'interprétation mazzinienne des écrits et de la pensée politique de Foscolo n'est pas sans créer des tensions entre l'exilé et les éditeurs florentins de l'œuvre du poète (Enrico Mayer, Gino Capponi et Pietro Bastogi) et enflamme le débat entre catholiques (représentés par Antonio Rosmini et Niccolò Tommaseo) et démocrates d'inspiration mazzinienne ${ }^{33}$. Dans une certaine mesure, la lecture de Mazzini compliqua la réception de Foscolo au cours du xix et créa l'image caricaturale du "foscolismo ${ }^{34}$ ».

Avant ses écrits politiques, Mazzini avait réussi à republier le Discorso sul testo del poema di Dante (I825) de Foscolo et à faire paraître pour la première fois le commentaire aux trois chants de la Commedia, laissé inachevé par le poète ${ }^{35}$. Dans les manuscrits de Foscolo, Mazzini n'avait trouvé que des notes relatives à l'Enfer, présentées sur des paperolles volantes, qui commentaient les variantes; les chants du Purgatoire et du Paradis étaient vierges. À part, figuraient aussi des index, des descriptions de manuscrits et d'imprimés et une chronologie, vraisemblablement de la main d'Antonio Panizzi. Pour réaliser le commentaire de la totalité du texte, Mazzini avait dû «immedesimarsi col suo metodo» :

A me intanto sembrava obbligo sacro verso Foscolo e la letteratura dantesca di non lasciare che andasse perduta la parte di lavoro compíta; e parevami di sentirmi capace di compirlo io stesso seguendo le norme additate da Foscolo nella correzione della prima cantica e immedesimandomi col suo metodo, l'unico, secondo me, che riscattando il poema dalla servitù alle influenze di municipio, toscane o friulane non monta, renda ad esso il suo carattere profondamente italiano ${ }^{36}$.

Mazzini réussit à convaincre l'éditeur Rolandi d'anticiper la somme conséquente demandée par Pickering (40o livres) et se mit au travail non pas pour compléter le commentaire, mais la présentation des variantes relatives au Purgatoire et au Paradis. Le résultat est l'ensemble de quatre volumes, dans une belle édition, philologiquement respectueuse de la volonté de Foscolo et présentée, dans la préface anonyme, comme un tournant dans l'histoire éditoriale de la Comédie. Le Discours qui précédait le commentaire évoquait en effet une solution éditoriale qui se voulait innovante, proposant explicitement un texte adapté à la sensibilité de

33. Cf. W. Binni, «Storia della critica foscoliana» (1957) dans Id., Ugo Foscolo. Storia e poesia, Turin, Einaudi, I982, p. 203-303.

34. Cf. C. Del Vento, "Quelques considérations sur la fortune historiographique de Foscolo», Chroniques italiennes, XVI, 2000, $\mathrm{n}^{\circ}$ 6I, p. 85-IO2.

35. Cf. P. Palmieri, «Il dantismo di Mazzini (tra Perticari e Foscolo)», Italianistica, 2006, n 3, p. 87-95; A. Bocchi, "Mazzini e il commento foscoliano alla "Commedia" ", Belfagor, 2007, p. 505-526; G. Federici, "L'edizione foscoliana della "Commedia" : Mazzini e Rolandi", Otto/Novecento, XXXII, 2008, n 3, p. I07-II6. 36. G. Mazzini, Note autobiografiche, ouvr. cité, p. 265-266. 
l'éditeur : le choix entre les très nombreuses variantes était résolu par l'empathie entre l'auteur originel et le commentateur moderne, lui aussi cultivé et poète. Le texte était en outre fondé sur le repérage d'un noyau expressif poétique original que Mazzini identifiait, conscient de son anachronisme, dans l'idée laïque de l'unité italienne.

Le travail de Mazzini fut accueilli avec peu d'enthousiasme de la part des admirateurs et amis de Foscolo, même si Mazzini avait recopié, en exergue à la Comédie, la lettre de Foscolo à son ami Capponi du 26 septembre I826 qui disait "Il Dante è libro da Italiani, e ch'io sempre intesi illustrarlo per l'Italia presente e futura $[. .$.$] A me, Gino mio, importa più$ ch'altro il non perdere tanti anni di studj intorno a Dante, ed al medio evo, e all'Italia ${ }^{37} »$.

Du point de vue philologique, la Commedia di Dante Alighieri illustrée par Ugo Foscolo n'était pas d'une valeur exceptionnelle, comme le reconnaissait Mazzini lui-même, dans une lettre à Quirina Mocenni Magiotti : «Non esagero a me né ad altri l'importanza del lavoro; mi pare una specie di tributo pagato alla memoria di Foscolo, e a questo ho anzi tutto pensato $^{38} »$. Dans tous les cas son but n'était pas philologique mais moral :

Il testo del Poema corretto da Foscolo è per me, letterariamente parlando, cosa abbastanza importante, perché si stampi. Ma la principale ragione che mi spronava ad accettare codeste noie del persuadere, del correggere e del curare siffatto lavoro, è morale: la vergogna dell'abbandonare ai tarli d'una bottega inglese, e dopo tanto cinguettare del «cantor de' Sepolcri» e della «illacrimata sepoltura» e di che no? il lavoro che costò ad Ugo la vita. Ho pensato che in Italia, dove si dànno quietamente cinquanta e più milioni di franchi all'austriaco, si potea spendere una somma d'alcune centinaia di lire per redimere quel lavoro. Ho pensato che, dov'anche il lavoro non valesse la somma, importava insegnare alla gioventù italiana il culto de' morti $[\ldots]^{39}$.

Son travail devait donc servir pour la jeunesse, pour lui apprendre le culte des morts, c'est pourquoi dès les premières pages, l'édition mazzinienne se présentait comme un prolongement de la leçon des Sepolcri ${ }^{40}$. La préface était précédée d'une gravure représentant le cimetière de Chiswick, et le préfacier déplorait «il giacersi dell'ossa di Foscolo in un cimitero straniero sotto una pietra postavi da mani straniere» qui justifiait pleinement le besoin de ne pas surcharger les souffrances de Foscolo avec l'oubli de la Commedia illustrata.

37. La "Commedia" di Dante Alighieri illustrata da Ugo Foscolo, Londres, Pietro Rolandi, 20 Berner's Street, I842.

38. G. Mazzini, A Q. Mocenni Magiotti (I8 avril I84I), dans Epistolario, Io; Scritti, ouvr. cité, vol. XX, p. I65.

39. G. Mazzini, A Q. Mocenni Magiotti (9 octobre I84I), ibid., p. 335-336.

40. Cf. S. Luzzatto, "Belli di fama e di sventura", art. cité, p. I42. 
Le commentaire foscolien eut une diffusion inférieure aux attentes, mais il inaugura une nouvelle saison dans la critique dantesque ${ }^{41}$ : en particulier, il traça la voie aux érudits qui se fondèrent sur une pratique de la critique attentive à la contextualisation historique et sensible au choix des mots, contre "le congetture avventate, le imposture letterarie, gli anacronismi eruditi, i mille errori accettati senza esame ${ }^{42}$ ", pour reprendre les mots de Mazzini. Mazzini toutefois avait en horreur les philologues et les controverses sur les variantes; il prônait plutôt une critique capable avant tout de recomposer "l'unità di un potente individuo, tipo di tutta una Nazione, grande e solenne di dolore ${ }^{43}$ » comme Dante. Mazzini sut surtout pointer la valeur historique du travail de Foscolo dans sa méthodologie : "Condusse la critica sulle vie della storia. Cercò in Dante non solamente il poeta, non solamente il padre della lingua nostra, ma il cittadino, il riformatore, l'apostolo religioso, il profeta della nazione ${ }^{44} »$.

Par son invitation à lire et à étudier Dante comme le génie qui préfigurait et guidait l'histoire, Mazzini transformait l'interprétation foscolienne, qui visait à répondre au "besoin d'apostolat religieux», en interprétation sociale et politique :

Oggi, pigmei, non intendiamo di Dante che il verso e la prepotente immaginazione; ma un giorno, quando saremo fatti più degni di lui, guardando indietro all'orme gigantesche ch'egli stampò sulle vie del pensiero sociale, andremo tutti in pellegrinaggio a Ravenna, a trarre dalla terra ove dormono le sue ossa gli auspicii delle sorti future e le forze necessarie a mantenersi su quell'altezza ch'egli, fin dal decimoquarto secolo, additava a' suoi fratelli di patria ${ }^{45}$.

Mazzini avait vu qu'avec Foscolo s'ouvrait le nouveau chapitre du dantisme romantique, pour lequel Dante s'élevait au-dessus de son époque, chargé d'idéaux et de missions atemporelles : "[Foscolo] riconobbe in Dante più che il poeta o il creatore della Lingua, il grande cittadino, il pensatore profondo, il Vate religioso, il profeta della nazionalità, dell'Italia ${ }^{46}{ }^{\prime}$.

Mis à part le caractère anachronique et généralisant, ce type de critique était capable d'obtenir de forts effets de suggestion. Dans ses essais, Mazzini forgeait un Dante à la figure d'apôtre, capable de toucher

4I. Cf. L. Russo, La nuova critica dantesca del Foscolo e del Mazzini e il suo valore politico, dans Id., Il tramonto del letterato. Scorci etico-politico-letterari sull'Otto e Novecento, Bari, Laterza, 1960, p. 187-212; ainsi que les pages que j’y ai consacrées dans mon récent volume Giuseppe Mazzini. Un intellettuale europeo, Naples, Liguori, 2013, p. $85-95$.

42. G. Mazzini, Commento foscoliano, art. cité, p. 46.

43. G. Mazzini, Opere minori di Dante (I844), dans Id., Scritti, ouvr. cité, vol. XXIX, p. 2 I4.

44. G. Mazzini, Commento foscoliano, art. cité, p. 46.

45. Ibid., p. 44.

46. G. Mazzini, Moto letterario, art. cité, p. 357. 
l'imagination et de mobiliser les Italiens. L'édition du commentaire foscolien participait au culte romantique de Dante, qui dans les années I830 et I840 s'était exprimé de différentes manières, de la prose politique au roman historique, et donna lieu à près de quarante éditions commentées de la Comédie. Le travail de Foscolo et Mazzini participait à un véritable genre littéraire, qui fixait les «gloires italiennes» de la patrie.

\section{Le mythe mazzinien du prophète en exil}

Les questions liées à l'interprétation mazzinienne de l'expérience de Foscolo nous ouvrent enfin sur de nouvelles pistes de recherche : l'histoire culturelle de l'exil risorgimental ${ }^{47}$. En effet, au cours du Risorgimento, l'exil cesse d'être simplement une expérience individuelle et prend la forme d'une véritable «institution» inaugurée par Foscolo, comme l'écrit Carlo Cattaneo ${ }^{4}$. Mazzini contribua largement à cette institution du mythe de l'exilé au cours du Risorgimento comme figure de l'italien, car si depuis les origines l'histoire de la littérature italienne est marquée par le thème de l'exil ${ }^{49}$, le $\mathrm{xIx}^{\mathrm{e}}$ siècle est véritablement «le siècle du mythe de l'exiléso".

Le "père de la patrie» Mazzini, «proscrit et étranger en Italie» («Italiano fuoruscito e straniero in Italia» pour reprendre la conclusion désenchantée à laquelle Foscolo était parvenue depuis l'époque de l'Ortis), développe dans ses essais et commentaires l'image risorgimentale de Foscolo comme prophète en exil, qui lui permet en retour d'acquérir une dignité de parole depuis l'étranger. L'exil devient un élément constitutif du mythe de la nation et comme un attribut nécessaire pour connoter le vrai patriote qui sacrifie son existence au projet national commun. Grâce à la propagande mazzinienne, qui récupère le personnage de Foscolo, dans la littérature risorgimentale et post-unitaire l'exilé a une immense fortune et devient une figure paradigmatique, chargée d'éléments symboliques : «una sorta

47. Sur ce thème, voir les contributions de A. Ciccarelli, Foscolo, Manzoni, and the Culture of Exile, dans A. Ciccarelli, P. A. Giordano (éd.), L'esilio come certezza. La ricerca d'identità culturale in Italia dalla rivoluzione francese ai nostri giorni, Bordighera, West Lafayette, 1998, p. 22-43; S. Tatti, Esuli e letterati: per una storia culturale dell'esilio risorgimentale, dans Q. Marini et alii (éd.), L'officina letteraria e culturale dell'età mazziniana (I815-1870). Giornate di Studio, Novi Ligure, Città del Silenzio, 2013, p. 89-I00; ainsi que ma synthèse "La nazione degli esuli del Risorgimento", dans N. di Nunzio, F. Ragni (éd.), "Già troppe volte esuli». Letteratura di frontiera e di esilio, t. I, Pérouse, Università degli Studi di Perugia, 20I4, p. I63-I79.

48. C. Cattaneo, «Ugo Foscolo e l'Italia» (I860), dans Id., Scritti letterari, P. Treves (éd.), Florence, I98I, vol. I, p. 536.

49. Cf. C. Dionisotti, Geografia e storia della letteratura italiana, Turin, Einaudi, 1967, p. 34; A. Asor Rosa, Genus italicum. Saggi sulla identità letteraria italiana nel corso del tempo, Turin, Einaudi, 1997, p. IO7-IIO.

50. G. De Marco, Mitografia dell'esule da Dante al Novecento, Naples, ESI, 1996. 
di proto-italiano, modello ideale di cittadino integerrimo, intriso di amor patrio, disposto al sacrificio ${ }^{5 \mathrm{I}}$.

Mazzini achève notamment son long itinéraire à travers l'œuvre de Foscolo au moment où Foscolo est soustrait à l'indigne sépulture du cimetière de Chiswick, dans les fumées de Londres, pour être rapatrié dans le sanctuaire de Santa Croce, après un long voyage en train à travers la Belgique, l'Allemagne et la Suisse. Mazzini, qui dans la dernière année de sa vie se déplaça sous fausse identité dans différentes villes italiennes, ne pouvait pas manquer de se rendre à Florence pour déposer une guirlande de fleurs sur la tombe de Foscolo, et y rédigea son dernier article sur le poète de l'exil. Le patriote propose un bilan de sa propre fidélité à Foscolo, depuis les premières émotions de sa vie, et en même temps un bilan de sa propre vie où il mesurait la distance entre ses espoirs passés et les déceptions présentes. La figure de Foscolo devenait un point de référence pour un discours sur la patrie comme Foscolo l'avait imaginée et comme l'avait projetée Mazzini. Tandis que le proscrit parcourait de nouveau les étapes de son exil, de l'Angleterre à la Suisse à l'Italie (toujours clandestin, surtout dans sa patrie), Mazzini sentait que l'accomplissement de son idéal était encore lointain. Son discours rejoignait le ton de l'invective emprunté à Foscolo, amère et émue :

E per la patria ch'io ideava intendo una patria d'uomini virtuosi e forti, onestamente alteri; puri nel pensiero e fedeli al pensiero nelle azioni; adoratori del Vero [...] che adorino Dio, la legge morale, la patria, l'umanità, il dovere temperato dall'amore, il sagrificio compíto con un sorriso. Era la Patria che Foscolo anch'egli sognava, per la quale ei patì persecuzioni, esilio, miseria e in seno alla quale avrebbe voluto aver tomba. L'abbiamo? No, non l'abbiamo ${ }^{2} \ldots$

Par un retour mimétique à l'éthique et au style des Dernières lettres de Jacopo Ortis, Mazzini, tout en saluant comme un geste patriotique la translation de la dépouille foscolienne vers Florence, mettait le doigt sur les incurables faiblesses de l'Italie unitaire, indigne de l'héritage transmis par les grands hommes de la trempe de Foscolos3.

Cette dernière rencontre entre Mazzini et Foscolo préfigure la continuité des destins des deux hommes, car comme le remarque Sergio Luzzato $^{54}$, les honneurs funèbres rendus au révolutionnaire génois, en

5I. S. Tatti, «Esuli e letterati», art. cité, p. 89.

52. G. Mazzini, Ugo Foscolo (I87I), dans Id., Scritti, ouvr. cité, vol. XCIV, p. 94-96.

53. Ces faiblesses furent également illustrées par la verve poétique du "jacobin" Carducci, dans son ode Per il trasporto delle reliquie di Ugo Foscolo in Santa Croce (24 giugno I87I).

54. S. Luzzatto, La mummia della repubblica. Storia di Mazzini imbalsamato, Turin, Einaudi, 20II, p. 23. 
mars I872, seront un calque de ceux organisés pour Foscolo à Santa Croce. Même si la proposition d'inhumer Mazzini dans l'église florentine est évidemment immédiatement écartée par la classe dirigeante du Royaume, sa dépouille sera, comme celle de Foscolo un an auparavant, transportée en train à travers les principales grandes villes d'Italie, et saluée par Carducci dans une composition qui reprend la même thématique de la dénonciation de la bassesse des vivants face à la grandeur des morts 55 .

\section{Conclusion}

Les funérailles des deux grands exilés du Risorgimento marquent aussi le passage à une nouvelle interprétation de l'œuvre de Foscolo dans l'Italie unifiée, celle de Francesco de Sanctis, qui dans un essai ensuite repris dans sa Storia della letteratura italiana redimensionnait la portée de la vie et de l'œuvre de l'auteur des Sepolcri. Le mythe du prophète en exil et du poète-soldat précurseur des premiers patriotes risorgimentaux est ainsi concurrencé par l'image du poète-libertin, dernier épigone des lettrés du xviII $^{\mathrm{e}}$ siècle ${ }^{56}$.

Dans le Royaume désormais définitivement achevé, où Rome avait retrouvé sa place de capitale, Foscolo et Mazzini en tant qu'icônes de l'exil comme «institution" n'avaient plus leur place : les rêves et les paroles abstraites des proscrits «perdants» furent étouffés par la nouvelle mythologie nationale des «vainqueurs" de la Troisième Italie.

55. G. Carducci, Per il passagio della salma di Giuseppe Mazzini, dans Opere, vol. IX, Edizione Nazionale, Bologne, Zanichelli, 1909, p. I3.

56. Cf. C. Dionisotti, "Foscolo esule» (198I), dans Id., Appunti sui moderni. Foscolo, Leopardi, Manzoni e altri, Bologne, il Mulino, I988, p. 55-78. 